\title{
Expanding biocatalysis for a sustainable future
}

\author{
Action for a more sustainable society must be taken - calling for advances in technology. This Insight features \\ artificial biocatalytic systems and functions not found in nature and emphasizes their potential to address major \\ challenges faced by humankind, such as climate change.
}

B iocatalysis is essential to life, as it drives important physiological reactions in all organisms.

Reactions such as photosynthesis and $\mathrm{N}_{2}$ fixation contribute to making the environment habitable for humans ${ }^{1}$. From ancient times, humankind took advantage of microorganisms to perform chemical transformations for their purposes. For example, the employment of yeast for the production of beer by fermentation can be dated back to the sixth millennium вС $^{2}$. However, it was only around the late 19th century that fermentation could be linked to enzymes produced by microorganisms ${ }^{3}$.

Since then, revolutionary advances have been made in biocatalysis, allowing humans to expand its application from natural fermentation processes to engineered biocatalytic systems involving isolated enzymes or whole cells with optimized functions and performances for the production of useful chemicals including medicinal drugs, polymers or biofuels ${ }^{4}$.

Now is a time in which another expansion of biocatalysis could play an important role in the further improvement of our lives and address some of the new challenges (many of them are self-inflicted) that human society is facing. We are producing huge amounts of waste such as plastics and greenhouse gases that harm the environment and threaten the public health and welfare of current and future generations. Helpful counteracting biocatalytic activities are provided by nature, such as photosynthesis for atmospheric $\mathrm{CO}_{2}$ fixation ${ }^{1}$. While nature has already developed large-scale approaches for certain problems, it can be a source of inspiration for others, like the hydrolysis of polyethylene terephthalate (PET) - the plastic from which many bottles are made ${ }^{5}$. However, these natural catalytic processes - as they stand - are apparently not sufficient to effectively mitigate the current challenges.

Recycling of waste and emissions is only one part of the solution - reducing them is the other. Engineered and hybrid biocatalytic systems could play an important role in addressing these areas.

This Insight centres on strategies to increase the performance and capabilities of biocatalytic systems with a major focus on their contribution to a more sustainable society. This includes the engineering of enzymes and microbial systems and their combination with other scientific disciplines, such as nanotechnology, materials science, photo- and electrochemistry.

\section{"Recycling of waste and emissions is only one part of the solution - reducing them is the other. Engineered and hybrid biocatalytic systems could play an important role in addressing these areas."}

In order to address the global challenges humankind faces, the countries of the United Nations adopted a set of goals designed to be "the blueprint to achieve a better and more sustainable future for all"'. In this Insight, Kristala Prather discusses the potential of engineered enzymes and organisms to contribute to addressing seven of the United Nations Sustainable Development Goals. On the quest to a more sustainable society it is important to replace energy-intensive and environmentally harmful reactions from the thermochemical industry with alternative processes. Biocatalytic systems usually operate at nearly ambient temperatures and pressures in an aqueous environment, without the production of toxic waste ${ }^{7}$. Unfortunately, many thermochemical transformations cannot be readily replaced by a biocatalytic alternative - oftentimes simply because there is no equivalent in nature. Impressively, over the past decade, employing directed evolution and the use of organic cofactors as well as other unnatural moieties, several such abiological transformations were added to the biocatalytic toolbox to fill this gap - these advances are summarized by Kai Chen and Frances Arnold. In addition, Ivana Drienovská and Gerard Roelfes specifically discuss the potential benefits of genetically encoding unnatural amino acids for this purpose.

Typically, designed enzymes contain only single catalytic active sites. In contrast, Zhi Zhou and Gerard Roelfes present an exciting Article, reporting that the introduction of two different abiological catalytic moieties in a single protein allows synergistic catalysis inaccessible to enzymes with a single catalytic site. Julia Sanz-Aparicio, Víctor Guallar, Manuel Ferrer and co-workers demonstrate that engineering two active sites with the same functionality in a single protein can increase the enzyme's overall turnover rate and reaction temperature range. These works indicate that the concept of installing multiple active sites in a single protein scaffold has potential for technological application, whereby it could possibly reduce isolation and purification steps and the amount of enzyme used reducing time, cost, waste and energy of the overall process.

The one-pot synthesis of products from sustainable starting materials under mild and environmentally friendly conditions is a worthwhile goal. Living cells can produce complex molecules from simple precursors by metabolic networks in a continuous and self-optimizing manner. Tobias Erb and colleagues discuss how these features could be introduced into man-made integrated catalytic systems for industrial synthesis with the aim to outcompete such naturally evolved metabolic networks. An important step to accomplish this is the spatial confinement of catalysts to create systems that can operate out of equilibrium with respect to their environment. In natural living systems this is achieved by membranebound compartments. Chemistry and materials science offer alternative solutions such as porous metal-organic framework nanoparticles or polymersomes for spatial confinement of catalysts. These and other technologies are discussed in a Review Article by Itamar Willner and colleagues to improve the performances of enzyme cascades. Luc Brunsveld, Tom de Greef and colleagues demonstrate that spatial localization using DNA nanotechnology can be used to design mimics of natural catalytic systems, allowing the interrogation of complex natural processes. 
Despite recent developments in chemistry and materials science, synthetic capacity and features such as self-replication of biological systems are so far hard to mimic. Indeed, microbial cell factories are a powerful tool to serve our needs in a sustainable and environmentally friendly manner ${ }^{8}$. However, to implement more sustainable bioprocesses in industry, economic viability plays an important role. In this respect, Liming Liu and co-workers present a strategy to improve the bioproduction of chemicals in Escherichia coli by engineering its lifespan. Such microorganisms can be tweaked to allow them to be fed with the greenhouse gas $\mathrm{CO}_{2}$ instead of sugar-based or biomass feedstocks for the production of chemicals. Advances in engineering microorganisms for this purpose are summarized by Tianwei Tan, Jens Nielsen and co-workers. Peidong Yang and co-workers review photosynthetic semiconductor biohybrids for the lightdriven catalytic conversion of $\mathrm{CO}_{2}$ to fuels and other value-added chemicals.
Thereby, the semiconducting nanomaterials provide stable components for solar energy capture, providing reducing equivalents for the microbial cells. Shelley Minteer and co-workers review the integration of electrodes to provide the microbial systems with the necessary reducing equivalents to drive biochemical reactions for the production of chemicals, fuels and materials using electricity that can be gained from renewable resources. Wolfgang Schuhmann and co-workers discuss the limitations of interfacing electrodes with isolated enzymes that need to be overcome for technological applications in energy conversion and solar energy harvesting. Although enzymes face stability issues in the latter set-ups, their mechanism, selectivity and reactivity might guide the further development of stable, high-performance bio-inspired systems to generate, store and transport electricity for a sustainable energy future.

This Insight highlights the manifold ways in which an expansion of biocatalysis can potentially improve our lives and lead to a more sustainable world for us and future generations. At the same time it underlines that many scientific disciplines are required to address the many current challenges in the world as best as possible. We hope that this Insight serves as an inspiration for the whole catalysis community to provide more and improved solutions, and wish you pleasant reading.

Published online: 18 March 2020 https://doi.org/10.1038/s41929-020-0447-8

References

1. Geider, R. J. et al. Glob. Chang. Biol. 7, 849-882 (2001).

2. Hartman, L. F. On Beer and Brewing Techniques in Ancient Mesopotamia (Read Books Ltd, 2013).

3. Lagerkvist, U. The Enigma of Ferment: From the Philosopher's Stone to the First Biochemical Nobel Prize (World Scientific, 2005).

4. Bornscheuer, U. T. et al. Nature 485, 185-194 (2012).

5. Yoshida, S. et al. Science 351, 1196-1199 (2016).

6. https://www.un.org/sustainabledevelopment/sustainabledevelopment-goals/

7. Burk, M. J. \& Van Dien, S. Trends Biotechnol. 34, 187-190 (2016). 8. Lee, S. Y. et al. Nat. Catal. 2, 18-33 (2019). 